\title{
Photoacoustic and thermoacoustic tomography of dog prostates
}

Haixin Ke, Zijian Guo, Todd N. Erpelding, Ladislav Jankovic, Robert L. Grubb, et al.

Haixin Ke, Zijian Guo, Todd N. Erpelding, Ladislav Jankovic, Robert L. Grubb, Lihong V. Wang, "Photoacoustic and thermoacoustic tomography of dog prostates," Proc. SPIE 7899, Photons Plus Ultrasound: Imaging and Sensing 2011, 789938 (22 February 2011); doi: 10.1117/12.875073

SPIE. Event: SPIE BiOS, 2011, San Francisco, California, United States 


\title{
Photoacoustic and thermoacoustic tomography of dog prostates
}

\author{
Haixin Ke ${ }^{*}$, Zijian Guo ${ }^{*}$, Todd N. Erpelding ${ }^{\mathrm{a}}$, Ladislav Jankovic ${ }^{\mathrm{a}}$, Robert L. Grubb III ${ }^{\mathrm{b}}$, Lihong V. \\ Wang* \\ *Optical Imaging Laboratory, Department of Biomedical Engineering, Washington University \\ 1 Brookings Drive, St. Louis, MO 63130, USA; \\ ${ }^{a}$ Philips Research North America, 345 Scarborough Road, Briarcliff Manor, NY 10510, USA; \\ ${ }^{b}$ Washington University School of Medicine, Division of Urology, 4960 Children's Place, St. Louis, \\ MO 63110, USA
}

\begin{abstract}
We developed a tri-modal system combining photoacoustic (PA) tomography, thermoacoustic (TA) tomography, and ultrasound (US) imaging. Acquired images of an excised dog prostate were compared to histology results. All three modalities can image distinct features. Features like the urethra were shown in both TA and US images, but TA gave a higher contrast-to-noise ratio. Fibrous tissue was more clearly imaged by TA, while the duct structure was better shown in PA images. These experimental results demonstrate the potential advantages of our tri-modal imaging system.
\end{abstract}

Keywords: Photoacoustic tomography, thermoacoustic tomography, prostate

\section{INTRODUCTION}

Prostate cancer is the most commonly diagnosed solid malignancy in men in the United States [1]. However, most tumors are small and many of these may not require aggressive treatment [2]. Current prostate cancer treatment options (surgical prostatectomy, radiation treatment, etc.) carry significant risk for urinary incontinence and sexual dysfunction. If urologists have the opportunity to treat only the tumors, this would be of great potential benefit to patients. Unfortunately, the ability to accurately localize all tumors in the prostate is poor and this limits the success of these treatments.

Current methods of prostate cancer imaging include transrectal ultrasound (TRUS), magnetic resonance imaging (MRI), computed tomography (CT), and near-infrared (NIR) diffuse optical tomography (DOT). However, these methods have limited ability to resolve malignant tissue and provide detailed information independently. For example, traditional TRUS lacks the necessary contrast and accuracy to identify most early stage tumors [3-5]. DOT provides good contrast of the blood-rich tumors but the resolution is not optimal because of the strong photon diffusion in biological tissues [6]. Using combinations of the methods may yield more accurate diagnoses and overcome the limited sensitivity and specificity [3].

Photoacoustic tomography (PAT) is a novel hybrid imaging technique that is noninvasive and has good contrast and good spatial resolution. The photoacoustic effects are based on the generation of pressure waves on absorption of electromagnetic energy [7]. The pressure generated is directly proportional to the local energy density absorbed in the tissue. Absorbed energy is converted into heat, which is further converted to a pressure rise via thermoelastic expansion. The initial pressure rise then propagates as ultrasonic waves, which are detected by ultrasound (US) sensors, and the received ultrasonic signals are used to form an image. In PAT, biological tissues are usually irradiated by a pulsed laser. When the excitation laser is replaced by microwave or radio-frequency (RF) sources, the technique is called thermoacoustic tomography (TAT) [8-10]. TAT/PAT combines high ultrasonic resolution and high contrast due to light or radio-frequency absorption $[11,12]$. They can reveal dielectric or optical properties of tissues that are closely related to the physiological and pathological status of the tissues [11]. This technique has been widely used in biomedical applications such as breast cancer imaging, brain structural and functional imaging, blood-oxygenation and hemoglobin monitoring, tumor angiogenesis.

PAT/TAT has the potential to detect subtle changes in tissue characteristics altered by the presence of tumors and to accurately image the tumors with high resolution. Although both rely on acoustic detection, TAT and PAT have different

Photons Plus Ultrasound: Imaging and Sensing 2011, edited by Alexander A. Oraevsky, Lihong V. Wang, Proc. of SPIE Vol. 7899, 789938 · C 2011 SPIE · CCC code: 1605-7422/11/\$18 · doi: 10.1117/12.875073 
contrast mechanisms. TAT measures dominantly water/sodium contrast while PAT measures blood volume and blood oxygenation contrast. Combining these modalities, as well as traditional US imaging techniques will provide complementary information regarding both the acoustic and optical properties of tumors; therefore improving the diagnosis and staging of prostate cancer by accurately localizing tumors within the prostate. Moreover, the integrated system is more efficient than the individual systems because three images can be acquired in the same setup without moving and realigning the sample [7].

We developed a multi-modality system by integrating PAT and TAT with a modified clinical ultrasound system. In this paper, the potential of this tri-modality imaging system was evaluated by imaging surgically excised healthy dog prostates. Although the prostate was from a healthy dog, the resulting PAT, TAT or US images identified some different areas of the prostate, conveying different tissue characteristics that were compared with histology results.

\section{METHOD}

TAT and PAT are compatible with ultrasonography because they can share the same ultrasound detection system. The multi-modality imaging system we developed, shown in Fig. 1, consists of a clinical ultrasound system, a laser source, a custom made microwave source, and the data acquisition system.

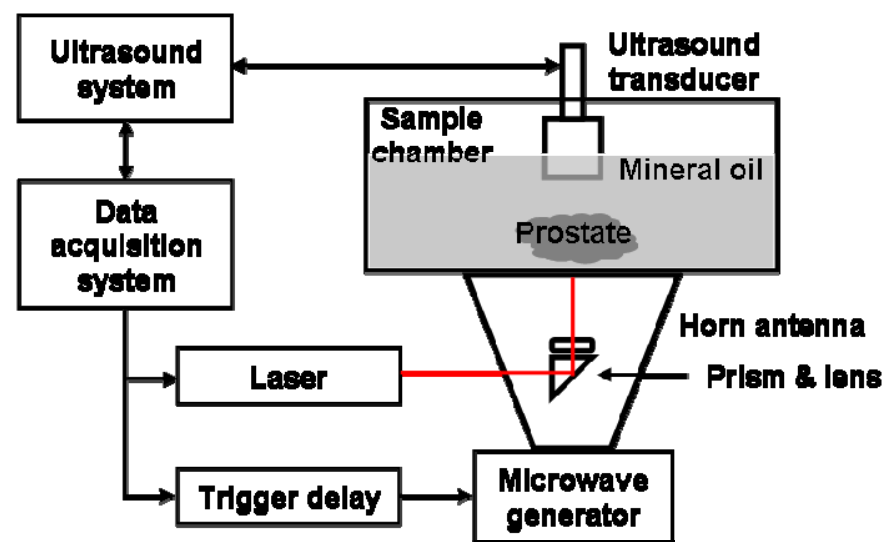

Figure 1. The main components of the system are the ultrasound system, the microwave system, the laser system, and the data acquisition system.

\subsection{Ultrasound system}

The modified clinical ultrasound imaging system (iU22, Philips Healthcare) can work independently and has been described in [13] and [14] previously. However, in the integrated system, the data acquisition system controls the triggering and the US data are transferred to the data acquisition system for display and post-processing. A US phased array transducer (S5-1, Philips Healthcare) with 80 elements and a nominal bandwidth of 1-5 MHz was used to detect US, PA and TA signals. The transducer can be positioned arbitrarily depending on which cross-section to image.

\subsection{Microwave source}

The microwave circuit generates $3 \mathrm{GHz}$ microwave pulses with different pulse widths and repetition rates and radiates them out through a horn antenna operating at its transverse electric (TE) mode $\mathrm{TE}_{10}$. The horn antenna has a small hole on the narrow side wall that allows for a laser coming into it. Since in $\mathrm{TE}_{10}$ mode the electrical field is parallel to the narrow side wall and approaches zero on the wall, the effect of the hole on the electromagnetic filed is minimized. In our experiments, the microwave operated at a repetition rate of $10 \mathrm{~Hz}$ with a pulse width of $0.3 \mu \mathrm{s}$.

\subsection{Laser source}

A tunable dye laser (PrecisionScan-P, Sirah), pumped by a Q-switched Nd:YAG laser (PRO-350-10, Newport), produces 6.5 -ns laser pulses at a repetition rate of $10 \mathrm{~Hz}$. The laser beam is guided into the horn antenna through a free space optical assembly. The light is expanded by a concave lens, homogenized by a ground glass and directed onto the 
prostate sample. The wavelength chosen for the experiment was $630 \mathrm{~nm}$. The incident laser fluence on the sample surface is controlled to be less than $20 \mathrm{~mJ} / \mathrm{cm}^{2}$, which is the American National Standards Institute limit [15].

\subsection{Data acquisition}

The data acquisition system integrates the above three parts together. It controls the triggering of US, laser, and microwave and collects three types of data: (1) the ultrasound system generates ultrasound pulse-echo data, (2) TAT data is generated using microwave excitation, and (3) PAT data is generated using light excitation. When working on the US mode, B-mode pulse-echo US images are acquired and the data are transferred to the data acquisition system. To obtain TA/PA data, the US transmitter is turned off and the US system works in receive-mode. Microwave and laser pulses are interleaved with a proper time delay, so that PA and TA data are acquired simultaneously at 10 frames per second, limited by the laser repletion rate. PAT, TAT and US images are then reconstructed using the delay-and-sum beam forming algorithm. With their different contrast mechanisms, we hope the three imaging modalities give us complementary information.

\subsection{Experimental procedures}

Our focus is to demonstrate that the multi-modality system has the ability to obtain comprehensive information of tissue characteristics. Specifically, we anticipate that US provides mainly structural information, while TAT differentiates areas with different water/sodium concentrations and PAT identifies physiological states such as blood volume and oxygen saturation.

The excised dog prostate was put in a plastic sample chamber. The chamber was filled with mineral oil which served primarily as a coupling medium for ultrasound propagation. The mineral oil was visibly transparent and did not absorb microwave so its effect on laser and microwave transmission was negligible. Laser and microwave illuminated the prostate from the bottom. The S5-1 ultrasound probe was placed in the mineral oil and above the sample with its axial axis perpendicular to the antenna opening. The prostate position was adjusted so that we imaged the cross-sections orthogonal to the urethra.

During the experiment, the relative position of the probe and the antenna as well as the laser beam was fixed. The chamber and the prostate sample were moved along the direction of the urethra using an optical stage, with a step size of $1.27 \mathrm{~mm}$. For each position, 2D pulse-echo US data was obtained first, followed by PA and TA data collected simultaneously. To improve the signal-to-noise ratio (SNR), the PA and TA acquisition was repeated 100 times and the raw data were averaged. PA and TA images were then reconstructed using a delay-and-sum beam-forming algorithm.

After imaging, the prostate was fixed immediately using formalin. Standard histology was performed by the small animal core of the Department of Pathology at Washington University. The histology slices were digitally photographed under a microscope. We then co-registered the photographs with our PAT/TAT/US images.

\section{RESULTS AND DISCUSSION}

The main body of the prostate gland is surrounded by a capsule and associated with striated urethralis muscle. The ductus deferentes run dorsally from the cranial prostate level and become the ejaculatory ducts piecing the body of the prostate. There is no duct at the caudal prostate level.

A total of 26 cross-section PAT/TAT/US images of the prostate and 9 histology slices along the direction of the urethra were acquired. Figure 2 (a) gives the photograph of the histology slice in the middle of the prostate gland. Figure 2 (b) shows the US image of this slice. The morphological features of the prostate such as the boundary of the prostate and the urethra (indicated by yellow arrow) can be recognized in the gray scale US image. Other than that, the prostate has a nearly uniform appearance in the US image because the sonographic characteristics of a healthy prostate are very homogeneous. It is worth noting that during the experiment, the prostate was a little deformed so the shape of the prostate on the images is not exactly the same as in the histology slices.

In Figure 3 (a), the acquired TAT image of the same cross-section was overlaid onto the US image. The urethralis muscle around the urethra gave very strong TA signal. The contrast was much better than that of the US image. Moreover, we can identify the fibrous tissue (the green arrow on bottom of the image) surrounding the ejaculatory ducts on the TAT image, by comparing to the photograph of the histology slide. Some of the fibro-muscular zone of the prostate (the green arrow on top of the image) was also visualized on TAT image. In Figure 3 (b), the acquired PAT image was overlaid onto the US image. It gave strong PA signal around the duct area. But we believe the strong contrast 
was given by the blood near the area. We had similar results for other slices. In conclusion, the US images showed some morphological features but the contrast was poor. The fibrous or muscular tissues were easily recognized on TAT images. The PA images showed the blood remaining in the prostate but did not provide much information inside the prostate because of the lack of contrast.

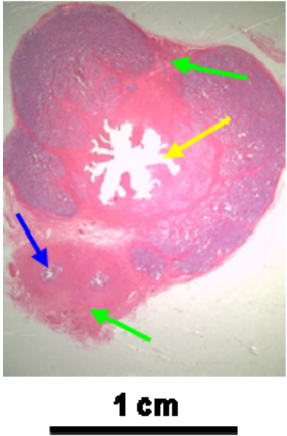

(a)

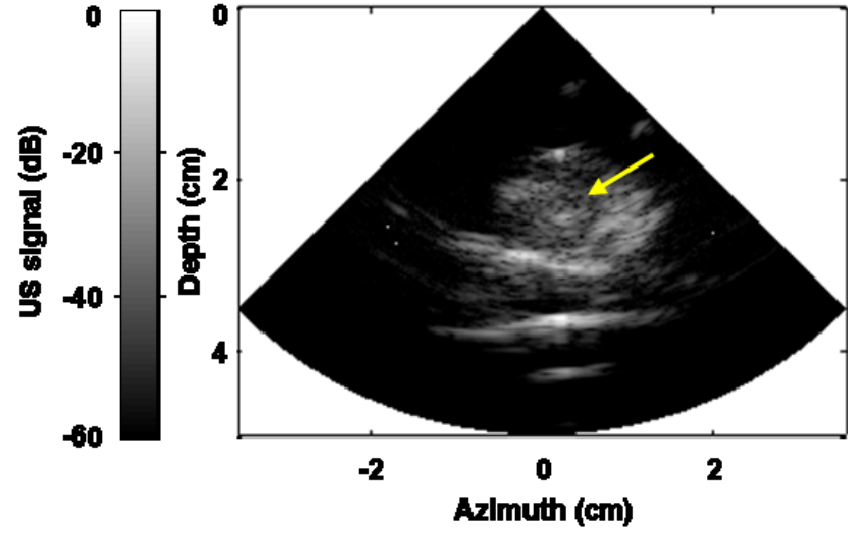

(b)

Figure 2. A photograph of the histology slice of the prostate and the corresponding B-mode US image. In (a), the histology slice, the yellow arrow indicates the urethra, the blue arrow indicates the ducts, and the green arrow indicates the fibro-muscular tissues. In (b), the US image, the urethra is recognized (yellow arrow).

\section{CONCLUSIONS}

We developed an imaging system which integrates three imaging modalities, namely, ultrasound, photoacoustic, and thermoacoustic tomography. The system added improved contrast and higher sensitivity provided by PAT/TAT to traditional US imaging techniques. This allows the multi-modality system to differentiate prostate tissues with different ultrasonic, dielectric, or optical properties at the same time. The feasibility of the integrated system was explored using an excised healthy dog prostate. We demonstrated that sonographically homogeneous structures which cannot be identified on US images can be more readily visualized on the TAT or PAT images. With the comprehensive information provided by the three modalities, the system may facilitate early detection of tumor, tumor localization, and staging of disease. 


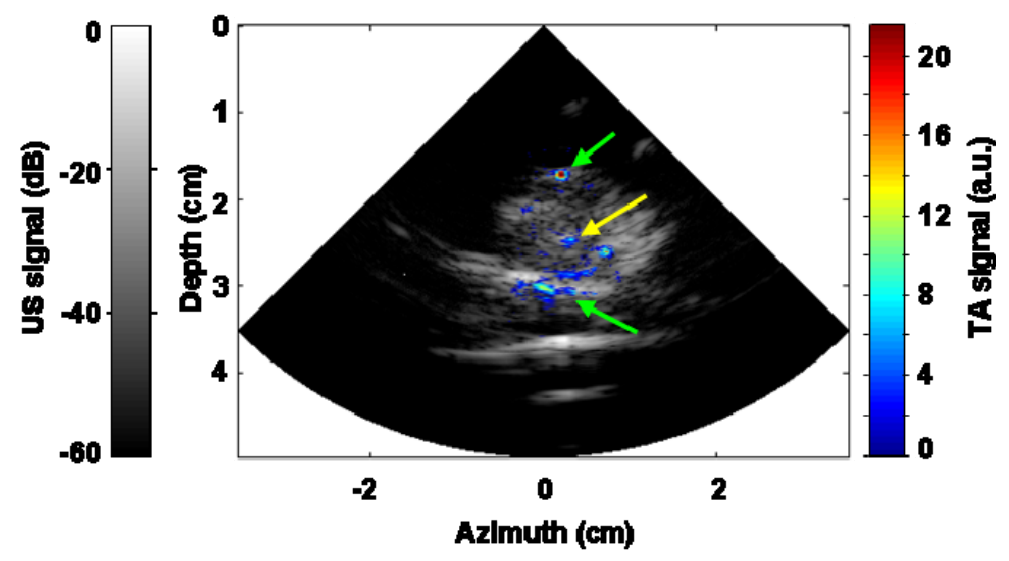

(a)

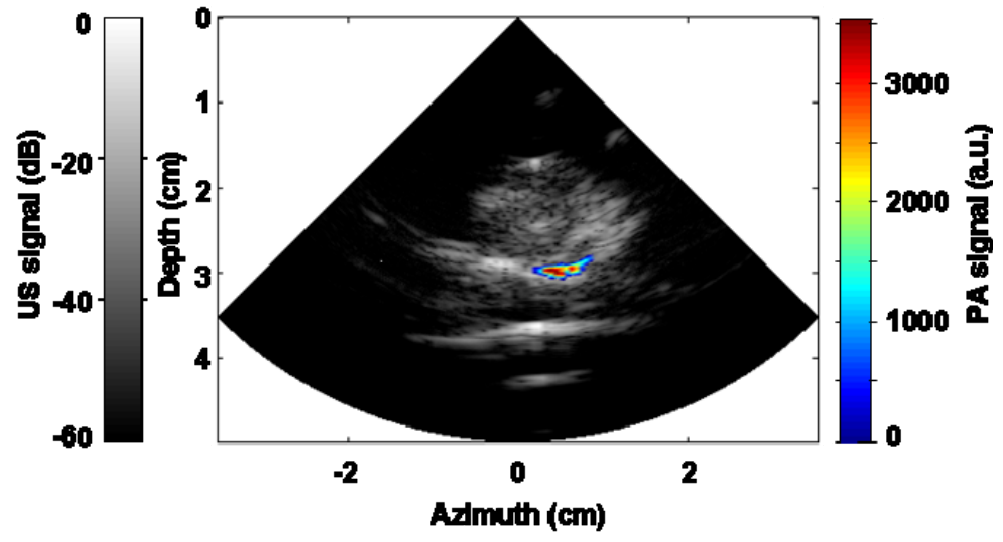

(b)

Figure 3. TA and PA images overlaid on US image. (a) The urethra (yellow arrow) was clearer on TA image; the fibro-muscular tissue around the ejaculatory ducts (green arrow on bottom) and on the boundary of the prostate (green arrow on top) also gave strong TA signal; (b) The PA signal was strong near the ducts, which was due to the blood in that area.

\section{REFERENCES}

[1] A. Jemal, R. Siegel, J. Xu, E Ward, “Cancer statistics, 2010,” CA: A Cancer Journal for Clinicians 60 (5), 277-300 (2010).

[2] L. Klotz, “Active surveillance for prostate cancer: for whom?” J. Clinical Oncology 23 (32), 8165-8169 (Nov. 2005).

[3] M Yaseen, S. A. Ermilov, H. Brecht, R. Su, A. Conjusteau, M. Fronheiser, B. A. Bell, M Motamedi, and A. A. Oraevsky. "Optoacoustic imaging of the prostate: development toward image-guided biopsy," J. Biomed. Opt. 15 (2), (2010).

[4] H. Wijkstra, M. H. Wink, and J. J. de la Rosette, "Contrast specific imaging in the detection and localization of prostate cancer," World J. Urol. 22 (5), 346-350 (2004).

[5] Z. Jiang, G. Reed Holyoak, K. E. Bartels, J.W. Ritchey, G. Xu, C. F. Bunting, G. Slobodov, J. S. Krasinki, and D. Piao, "In vivo trans-rectal ultrasound coupled trans-rectal near-infrared optical tomography of canine prostate bearing transmissible venereal tumor," in Optical Tomography and Spectroscopy of Tissue VIII, B. J. Tromberg, A. G. Yodh, M. Tamura, E. M. Sevick-Muraca, and R. R. Alfano, Proc. SPIE 7174, 71741U (2009). 
[6] X. Wang, W. W. Roberts, P. L. Carson, D. P. Wood, and J. B. Fowlkes, "Photoacoustic tomography: a potential new tool for prostate cancer," Biomedical Optics Express 1 (4), 1118-1126 (2010).

[7] M. Pramanik, G. Ku, C. H. Li, and L. V. Wang, "Design and evaluation of a novel breast cancer detection system combining both thermoacoustic (TA) and photoacoustic (PA) tomography," Medical Physics 35, 2218-2223 (2008).

[8] Kruger, R. A., Reinecke, D. R.,Kruger, G. A., "Thermoacoustic computed tomography-technical considerations," Medical Physics 26 (9), 1832-1837 (1999).

[9] Wang, L. H. V., Zhao, X., Sun, H.,Ku, G., "Microwave-induced acoustic imaging of biological tissues," Review of Scientific Instruments 70 (9), 3744-3748 (1999).

[10] M. Xu and L. V. Wang, "Photoacoustic imaging in biomedicine," Review of Scientific Instruments 77, 041101-(122) (2006).

[11] G. Ku, B. D. Fornage, X. Jin, M. Xu, K. K. Hunt, and L. V. Wang, "Thermoacoustic and photoacoustic tomography of thick biological tissues toward breast imaging," Technology in Cancer Research and Treatment 4 (5), 559-566 (2005).

[12] L. V. Wang, “Prospects of photoacoustic tomography,” Medical Physics 35 (12), 5758-5767 (2008).

[13] T. N. Erpelding, C. Kim, M. Pramanik, L. Jankovic, K. Maslov, Z. Guo, J. A. Margenthaler, M. D. Pashley, and L. V. Wang, "Sentinel lymph nodes in the rat: noninvasive photoacoustic and US imaging with a clinical US system," Radiology 265 (1), 102-110 (2010).

[14] C. H. Kim, T. N. Erpelding, L. Jankovic, and L. V. Wang, "Performance benchmarks of an array-based hand-held photoacoustic probe adapted from a clinical ultrasound system," in press.

[15] Laser Institute of America, American National Standard for Safe Use of Lasers ANSI Z136.1-2000, American National Standards Institute, Inc., New York, NY, 2000. 